\title{
Comparison of the Microsatellite and Single Nucleotide Polymorphism Methods for Discriminating among Hanwoo (Korean Native Cattle), Imported, and Crossbred Beef in Korea
}

\author{
Eun-Jeong $\mathrm{Heo}^{1}$, Eun-Kyung Ko, Kun-Ho Seo${ }^{2}$, Jung-Whan Chon², Young-Jo Kim², \\ Hyun-Jung Park ${ }^{1}$, Sung-Hwan Wee, and Jin-San Moon* \\ Animal and Plant Quarantine Agency, Anyang 430-757, Korea \\ ${ }^{1}$ Ministry of Food and Drug Safety, Cheongwon 363-951, Korea \\ ${ }^{2}$ KU Center for Food Safety, College of Veterinary Medicine, Konkuk University, Seoul 143-701, Korea
}

\begin{abstract}
The identity of 45 Hanwo and 47 imported beef (non-Hanwoo) samples from USA and Australia were verified using the microsatellite (MS) marker and single nucleotide polymorphism (SNP) methods. Samples were collected from 19 supermarkets located in the city of Seoul and Gyeonggi province, South Korea, from 2009 to 2011. As a result, we obtained a $100 \%$ concordance rate between the MS and SNP methods for identifying Hanwoo and non-Hanwoo beef. The MS method presented a 95\% higher individual discriminating value for Hanwoo (97.8\%) than for non-Hanwoo (61.7\%) beef. For further comparison of the MS and SNP methods, blood samples were collected and tested from 54 Hanwoo $\times$ Holstein crossbred cattle (first, second, and third generations). By using the SNP and MS methods, we correctly identified all of the firstgeneration crossbred cattle as non-Hanwoo; in addition, among the second and third generation crossbreds, the ratio identified as Hanwoo was $20 \%$ and $10 \%$, respectively. The MS method used in our study provides more information, but requires sophisticated techniques during each experimental process. By contrast, the SNP method is simple and has a lower error rate. Our results suggest that the MS and SNP methods are useful for discriminating Hanwoo from non-Hanwoo breeds.
\end{abstract}

Keywords: Hanwoo, genetic identification, microsatellite method, single nucleotide polymorphism method

\section{Introduction}

Korean native cattle (Hanwoo) have been raised in the Korean Peninsula since 2000 B.C. The specific traits of Hanwoo have been maintained through careful breeding, and therefore the current blood lineage is very valuable and pure (Kim and Lee, 2000; Lim et al., 2014). In 2011, approximately 3 million heads of beef cattle were raised in Korea. In the same year, the total number of slaughtered cattle was 851,000 , including 720,000 Korean native cattle and 131,000 domestic Holsteins. Nevertheless, Korea is only a $42.8 \%$ self-sufficient nation in the beef market; the country imports the rest of Korean beef from Australia, the USA, New Zealand, Mexico, and Canada, making Korea the fourth-largest global importer of beef (Korea Meat Trade Association, 2011).

\footnotetext{
*Corresponding author: Jin-San Moon, Veterinary Pharmaceutical Management Division, Animal and Plant Quarantine Agency, Anyang 430-757, Korea. Tel: +82-31-467-4303, Fax: +8231-467-4321, E-mail: moonjs727@korea.kr
}

Domestic meat consumption is increasing in Korea, because of a steady expansion in the proportion of Koreans eating out, and this is leading to a rise in imports of livestock products (Min et al., 1995). The Uruguay Round, in particular, has accelerated the opening of markets to imports of livestock products; further, the signing of the Free Trade Agreement (FTA) has fully opened doors to large quantities of imported beef. Meanwhile, consumer anxiety regarding imported livestock products is rising because of the occurrences of bovine spongiform encephalopathy (BSE) in countries such as the USA, Canada, Israel, and Japan (Hwang, 2010). Imported beef may be mislabeled as Hanwoo, based on the higher price it commands, and unscrupulous companies are taking advantage of consumers' increasing concerns regarding the safety of imported beef (Cheong et al., 2013).

Korean consumers' preference for Hanwoo is increasing; however, there has been a rise in the number of cases of restaurants selling imported beef and domestic Holstein beef, which are relatively low in cost, as Hanwoo (Hong et al., 2010). Hence, in August 2008, the Korean 
government implemented a system for labeling beef by country of origin, as part of safety measures designed to distinguish Hanwoo from imported beef. On 22 December 2008, the Animal and Meat Traceability System for Hanwoo put these rules into practice. Additionally, the Law of Labeling the Origin of Meat in Restaurants was implemented to protect the rights of consumers by providing accurate information on the origins of meat products (Hwang, 2010).

Given these circumstances, breed determination based on scientific methods is crucial, in order to provide consumers with accurate information about beef products. Conventionally, cattle breeds are identified by means of physical attributes, such as horns, coat color, and frame. However, in the case of seasoned or processed beef, it is difficult to distinguish products by means of visual inspection; hence, the need for scientific methods such as gene analysis has been raised (Chung and Chung, 2004; Hong et al., 2010). To fulfill the need for a scientific breed identification method based on gene analysis, PCR-single-strand conformation polymorphism by using the melanocortin 1 receptor (MC 1R) gene, which determines cattle coat color, was developed in 2000 (Berryers et al., 2003; Chung et al., 2000; Chung and Chung, 2004; Cone et al., 1996; Do et al., 2007). Subsequently, the MC1R PCR-RFLP (restriction fragment length polymorphism) marker was used for discrimination of beef origin (Min et al., 1995).

However, the MC1R method alone cannot easily discriminate between domestic Hanwoo beef and imported beef from animals with a genealogy of light brown coat color (Cheong et al., 2013; Mohanty et al., 2008). As such, methods based on other DNA markers such as microsatellite (MS) markers - recommended by the International Society of Animal Genetics (ISAG) for analysis of polymorphism in cattle - are widely used in Hanwoo traceability research (Lee et al., 2004; Lee et al., 2008; Lim et al., 2005; Yoon et al., 2005). Currently, single nucleotide polymorphism (SNP) markers, which were found in large numbers through decoding of the entire nucleotide sequence of the bovine chromosome, are distributed in a readily usable DNA chip form (Ball et al., 2010; Sasazaki et al., 2011; Van Eenennaam et al., 2007).

In 2008 and 2010, respectively, the MS (Lim et al., 2009) and SNP (Cheong et al., 2013) methods were developed domestically as certified testing methods for verifying Hanwoo. However, the efficacies and accuracies of these two methods have not previously been evaluated objectively. For example, the MS method's standard probabil- ity is currently set at 0.5 for identifying Hanwoo and nonHanwoo beef (MFDS, 2013); this value is too low, which increases the chance of mislabeling crossbred cattle as Hanwoo. In addition, the tests require to be validated, including a comparison in terms of accuracy. In the present study, we verified the identity of 45 native beef samples (Hanwoo) and 47 imported beef samples (non-Hanwoo), by using the MS and SNP methods. In addition, we conducted a comparison analysis of the agreement rates between the two methods for meat and blood samples from Hanwoo, imported beef, and Hanwoo-Holstein crossbred animals in Korea.

\section{Materials and Methods}

\section{Samples}

We collected 45 beef samples labeled as Hanwoo and 47 beef samples labeled as non-Hanwoo (including domestic Holstein and imported beef from the USA and Australia) of beef portion such as tender loin, sirloin, chuck roll and loin from 19 major supermarket chains and department stores located in Seoul city and Gyeonggi province, South Korea, from 2009 to 2011. We applied the MS and SNP methods to all of the samples, to discriminate their origin. In order to evaluate the accuracy of identifying crossbred cattle by using the MS and SNP methods, we tested blood samples collected from 54 Hanwoo $\times$ Holstein crossbred cattle (44 first-generation, and 10 second- and third-generation) on individual cow farms in Korea.

\section{DNA extraction from samples}

DNA extraction was conducted in accordance with the Hanwoo verification test method specified by the Standards for Processing and Ingredient Specifications of Livestock Products of the Ministry of Food and Drug Safety (MFDS, 2013). Briefly, $1 \mathrm{~g}$ of a test sample was diced into fine pieces and added to $5 \mathrm{~mL}$ of SPK buffer ( 50 of $54 \%$ sucrose, $2 \mathrm{~mL}$ of $50 \mathrm{mM}$ ethylene diamine tetraacetic acid [EDTA], $10 \mathrm{~mL}$ of $10 \% \mathrm{SDS}, 5 \mathrm{~mL}$ of $20 \times \mathrm{SSC}$, and $33 \mathrm{~mL}$ of distilled water), $2 \mu \mathrm{L}$ of RNaseA (Qiagen, USA), and $5 \mu \mathrm{L}$ of Proteinase K (Qiagen, USA), and then inverted. The sample was then placed in a $55^{\circ} \mathrm{C}$ water bath overnight, mixed with $3 \mathrm{M} \mathrm{NaCl}$ solution $(500 \mu \mathrm{L})$, inverted, and then centrifuged for $15 \mathrm{~min}$ at $3000 \mathrm{rpm}$. The DNA pellet was transferred to a 1.1-mL Eppendorf (EP) tube, mixed with $70 \% \mathrm{EtOH}(1 \mathrm{~mL})$, inverted, and then centrifuged for $1 \mathrm{~min}$ at $13000 \mathrm{rpm}$. After removing the supernatant, the DNA in pellet form was collected 
and dried in an oven. Finally, TE buffer $(150 \mu \mathrm{L})$ was added to the dried DNA pellet to produce the DNA test sample.

\section{Microsatellite method}

We used the MS method with 48 markers for identifying Hanwoo and non- Hanwoo cattle, in accordance with the Standards for Processing and Ingredient Specifications of Livestock Products (Kim et al., 2009; MFDS, 2013). Briefly, the PCR reaction mixture contained $2 \mathrm{~mL}$ of genomic DNA (25-50 ng) test sample, PCR primer mix $(8.25 \mu \mathrm{L}), 10 \times$ reaction buffer $(1.5 \mu \mathrm{L})$, dNTPs $(1.2$ $\mu \mathrm{L}), \mathrm{MgCl}_{2}(1.1 \mu \mathrm{L}), 0.4 \mu \mathrm{L}$ of Taq DNA polymerase (1$2 \mathrm{U})$, DMSO $(0.25 \mu \mathrm{L})$, and deionized water $(0.3 \mu \mathrm{L})$. We used triplicate $(15-\mu \mathrm{L})$ sets of primer mix (SET 0 , SET 1 , SET 2) for each reaction. For PCR analysis, denaturation was conducted for 25 repeating cycles. The first 5 cycles each consisted of $4 \mathrm{~min}$ at $95^{\circ} \mathrm{C}, 60 \mathrm{~s}$ at $94^{\circ} \mathrm{C}, 75 \mathrm{~s}$ at $58^{\circ} \mathrm{C}$, and $60 \mathrm{~s}$ at $72^{\circ} \mathrm{C}$. The next 5 cycles each consisted of $60 \mathrm{~s}$ at $94^{\circ} \mathrm{C}, 75 \mathrm{~s}$ at $57^{\circ} \mathrm{C}$, and $60 \mathrm{~s}$ at $72^{\circ} \mathrm{C}$. The final 5 cycles each consisted of $60 \mathrm{~s}$ at $94^{\circ} \mathrm{C}, 75 \mathrm{~s}$ at $56^{\circ} \mathrm{C}$, and $60 \mathrm{~s}$ at $72^{\circ} \mathrm{C}$. Finally, a 30-min extension step was performed at $65^{\circ} \mathrm{C}$, to conclude the cycle at $8^{\circ} \mathrm{C}$. The PCR products $(1 \mu \mathrm{L}$ ) were diluted with distilled water (SET 0 , 60 times; SET 1, 40 times; SET 2, 60 times) and used for allelic analysis. To $1 \mu \mathrm{L}$ of the diluted PCR product sample, $10 \mu \mathrm{L}$ of Hi-Di Formamide ${ }^{\mathrm{TM}}$ (Applied Biosystems, $\mathrm{UK}), 1 \mu \mathrm{L}$ of size marker (GeneScan ${ }^{\mathrm{TM}}-500 \mathrm{LIZ}^{\circledR}$ size standard, Applied Biosystems, UK) were added, and the mixture was then divided into 96-well plates. These 96well plate mixtures were allowed to react at $95^{\circ} \mathrm{C}$ for 3 min, and were then immediately cooled to a temperature of $4^{\circ} \mathrm{C}$. The PCR products were analyzed by using an ABI 3130xl Genetic Analyzer (Applied Biosystems), and the exact size of alleles was determined by using GeneMapper Software v.4.0 (Applied Biosystems). The identification decision was made by applying the breed assignment method of Rannala and Mountain (1997), based on the relative probability of a sample's assignment to the 2 groups, namely, Hanwoo or non-Hanwoo. The sample was identified as belonging to 1 of the 2 groups when the probability was $\geq 0.5$.

\section{Single nucleotide polymorphism method}

We conducted the SNP method, which uses 90 markers for identifying Hanwoo/crossbred/non-Hanwoo cattle, in accordance with the Standards for Processing and Ingredient Specifications of Livestock Products (Cheong et al., 2013; MFDS, 2013). DNA samples were used at a con- centration of $50 \mathrm{ng} / \mu \mathrm{L}$. For discrimination marker genotyping, $5 \mu \mathrm{L}$ of DNA plate production reagent were added to DNA plates. The DNA samples were transferred to the plates, and were allowed to react according to the manufacturer's instructions. Next, $10 \mu \mathrm{L}$ of the DNA reaction mixture were added to genotype-specific extension reaction plates, and mixed until the beads were completely dispersed. Pooled-sampling lengthening and pairing reactions of beads with 90 markers were then conducted. For PCR analysis, $64 \mu \mathrm{L}$ of DNA polymerase and $50 \mu \mathrm{L}$ of uracil DNA glucosylase were added to the PCR mixture solution tube and thoroughly mixed. Next, $30 \mu \mathrm{L}$ aliquots of the mixture were placed on PCR plates, and $35 \mu \mathrm{L}$ of DNA were added to each genotype-specific extension reaction containing beads. PCR analysis was conducted over 34 cycles, consisting of $10 \mathrm{~min}$ of denaturation at $37^{\circ} \mathrm{C}$ and $3 \mathrm{~min}$ of denaturation at $95^{\circ} \mathrm{C}$, followed by $35 \mathrm{~s}$ at $95^{\circ} \mathrm{C}, 35 \mathrm{~s}$ at $56^{\circ} \mathrm{C}$, and $2 \mathrm{~min}$ at $72^{\circ} \mathrm{C}$, and a final extension step of $10 \mathrm{~min}$ at $72^{\circ} \mathrm{C}$. The VeraCode bead plates completed through PCR reactant combination process and VeraCode bead plates compound the reaction, were scanned by using BeadXpress reader (Illumina, USA). On completion of scanning, genotyping was determined by using bead studio software. The final genotype file was analyzed automatically by using the Hanwoo discrimination formula of MFDS. The object was determined as Hanwoo if the estimated value was $\leq 0.45$ and as nonHanwoo if the estimated value was $>0.45$.

\section{Results and Discussion}

\section{Monitoring the labeling of Hanwoo beef and im- ported beef by using the MS and SNP methods}

On December 22, 2008, the Korean government implemented laws governing cattle and beef traceability, as part of safety measures designed to distinguish Hanwoo from imported beef (Hwang, 2010). Currently, the MS and SNP methods are used domestically as certified testing methods for verifying Hanwoo (MFDS, 2013). In the present study, we verified the identity of 45 native beef samples (Hanwoo) and 47 imported beef samples (non-Hanwoo; domestic Holstein meat and imports from the USA and Australia), by using the MS and SNP methods. We obtained a $100 \%$ concordance rate for beef labeling between the 2 methods for all of the 92 beef samples (Table 1). In the case of the MS method for Hanwoo beef, 44 samples $(97.8 \%)$ had a probability value of $\geq 95.0 \%$, and only 1 sample (2.2\%) showed a probability value of $85.0-94.9 \%$ (Table 2). On the other hand, for non-Hanwoo beef, 29 
Table 1. Comparison between microsatellite (MS) and single nucleotide polymorphism (SNP) methods for discriminating Hanwoo and non-Hanwoo-labeled beef obtained from supermarkets in Korea

\begin{tabular}{cccccc}
\hline \hline \multirow{2}{*}{ Classification } & \multicolumn{2}{c}{ MS method } & \multicolumn{2}{c}{ SNP method } \\
\cline { 2 - 6 } & & Hanwoo & Non-Hanwoo & Hanwoo & Non-Hanwoo \\
\cline { 2 - 6 } & Hanwoo $(\mathrm{n}=45)$ & 45 & 0 & 45 & 0 \\
\hline No. of samples & 0 & 47 & 0 & 47 \\
tested $(\mathrm{n}=92)$ & Non-Hanwoo $(\mathrm{n}=47)$ & $100 \%$ & $100 \%$ & $100 \%$ & $100 \%$ \\
\hline \multicolumn{2}{c}{ Positive ratio for label } & &
\end{tabular}

Table 2. Analysis of discriminating values for Hanwoo and non-Hanwoo-labeled beef by using the microsatellite (MS) method

\begin{tabular}{ccccccc}
\hline \hline \multirow{2}{*}{ Breeds } & \multirow{2}{*}{ No. of beef samples (\%) } & \multicolumn{5}{c}{ Individual discriminating value (\%) } \\
\cline { 3 - 7 } & & $<50$ & $50-74.9$ & $75-84.9$ & $85-94.9$ & $\geq 95$ \\
\hline Hanwoo & $45(100)$ & $0(0)$ & $0(0)$ & $0(0)$ & $1(2.2)$ & $44(97.8)$ \\
Non-Hanwoo & $47(100)$ & $1(2.1)$ & $8(17.0)$ & $4(8.5)$ & $5(10.6)$ & $29(61.7)$ \\
\hline Total & $92(100)$ & $1(1.1)$ & $8(8.7)$ & $4(4.4)$ & $6(6.5)$ & $73(79.4)$ \\
\hline
\end{tabular}

samples (61.7\%) had a probability value of $\geq 95.0 \%, 5$ samples $(11.6 \%)$ showed probability values of 85.0 $94.9 \%$, 4 samples $(9.3 \%)$ showed probability values of $75-84.9 \%, 8$ samples $(18.6 \%)$ showed probability values of $50-74.9 \%$, and only 1 sample $(2.3 \%)$ showed a probability value of $<50 \%$. In the case of MS method, Hanwoo respresented higher overall probability rates compared to non-Hanwoo with all samples showing probability rates of $85 \%$ and higher. This result could be due to difference in sample that Hanwoo was relatively purebred Korean native cattle in contrast with non-Hanwo (imported beef). However, despite the small differences in the probability values between Hanwoo and non-Hanwoo beef, our results showed a $100 \%$ concordance rate for labeled beef. We subsequently identified the test samples by comparing the results of our present analysis with the reference genotype database of samples that had previously been identified as Hanwoo or non-Hanwoo by using the SNP method (Cheong et al., 2013; MFDS, 2013). The results of previous analyses of test samples (Hanwoo, crossbred, and pure non-Hanwoo beef) by using the SNP method showed that the SNP values ranged $0.30-0.45$ for Hanwoo beef, 0.80-1.34 for crossbred beef, and 1.60-2.24 for pure non-Hanwoo beef (Cheong et al., 2013; MFDS, 2013). Accordingly, the results of our present study identified samples with discrimination values of 0.45 as Hanwoo, and samples with discrimination values of $>0.45$ as non-Hanwoo beef (data not shown). The results of the SNP method showed a $100 \%$ concordance rate for country of origin of beef labeling for all the 92 beef samples. Thus, we obtained a $100 \%$ concordance rate between the MS and SNP methods for Hanwoo and non-Hanwoolabeled beef.

\section{Concordance rate between the MS and SNP meth-} ods for crossbred Hanwoo cattle and Holstein cattle

In Korea, crossbred cattle are limited to Hanwoo and Holstein crossbreeds. The reason is the specificity of the country's livestock industry in Korea, with the Korean government allowing only Hanwoo and Holstein sires to be used in breeding projects or in the domestic beef industry (Cheong et al., 2013). Therefore, in our present comparison study, we used only Hanwoo and Holstein crossbreeds.

We investigated the concordance rate between the MS and SNP methods conducted on blood samples of 54 crossbred cattle, consisting of first-, second-, and thirdgeneration Hanwoo $\times$ Holstein crossbred cattle (Table 3). Our results showed that the two methods correctly identified first-generation crossbred cattle as non-Hanwoo with $100 \%$ accuracy; however, both methods were less likely correctly to identify second- and third-generation crossbred cattle - showing the appearance of Hanwoo - as nonHanwoo (accuracies of $80.0 \%$ and $90.0 \%$ for the MS and SNP methods, respectively). All of the samples that were incorrectly identified as Hanwoo by using the two methods from second- and third-generation crossbred cattle.

According to the current Hanwoo verification test procedure of Standards for Processing and Ingredient Specifications of Livestock Products, a probability value of $\geq 50 \%$ determined by using the MS method is sufficient to classify a sample as Hanwoo (MFDS, 2013). However, in our present study, a probability value of $\geq 85 \%$ showed a higher accuracy for Hanwoo discrimination. Thus, in order to increase accuracy of Hanwoo discrimination, the probability value criterion should be adjusted to $\geq 75 \%$. In fact, a large number of third-generation Holstein $\times$ Hanwoo crossbreds are phenotypically identifiable as Hanwoo. 
Table 3. Concordance rate between microsatellite (MS) and single nucleotide polymorphism (SNP) methods used for analysis of blood samples obtained from crossbred Hanwoo and Holstein cattle

\begin{tabular}{|c|c|c|c|c|c|c|c|}
\hline \multirow{2}{*}{$\begin{array}{c}\text { Crossbred } \\
(\text { Hanwoo } \times \text { Holstein })\end{array}$} & \multirow{2}{*}{$\begin{array}{l}\text { No. of blood } \\
\text { samples }\end{array}$} & \multicolumn{3}{|c|}{ MS method } & \multicolumn{3}{|c|}{ SNP method } \\
\hline & & Hanwoo & Non-Hanwoo & accuracy & Hanwoo & Non-Hanwoo & accuracy \\
\hline F1 & 44 & 0 & 44 & $100 \%$ & 0 & 44 & $100 \%$ \\
\hline $\mathrm{F} 2 \& \mathrm{~F} 3$ & 10 & 2 & 8 & $80.0 \%$ & 1 & 9 & $90.0 \%$ \\
\hline Total & 54 & 2 & 52 & $96.3 \%$ & 1 & 53 & $98.1 \%$ \\
\hline
\end{tabular}

Therefore, it is likely that third and higher generations of crossbred cattle have a higher probability of genetically being identified as Hanwoo. Further studies to discriminate Hanwoo in the next generation of various crossbred cattle are required.

Microsatellites have been widely used for genetic characterization of beef origin and the study quantitative trait loci (Chung and chung 2004; Oh et al., 2008). However, they may provide misleading results with higher error rates than those of SNPs (Ball et al., 2010; Cheong et al., 2013; Van Eenennaam et al., 2007). In comparison with the SNP methods, the MS method is more informative. However, the SNP method is simple and has a lower error rate (Cheong et al., 2013). Our present results indicate that, if the discrimination value of the MS method is corrected, the SNP and MS methods may be usefully combined with specific Hanwoo DNA markers, to detect violations of the country of origin labeling law. Given the increased imports of beef in Korea, more intensive evaluation of Hanwoo discriminating methods, and also the construction of a national beef DNA database are required.

\section{Acknowledgements}

This study was supported by the Animal and Plant Quarantine Agency's veterinary science technology development and research project (N-FS03-2011-11-01).

\section{References}

1. Ball, A. D., Stapley, J., Dawson, D. A., Birkhead, T. R., Burke, T., and Slate, J. (2010). A comparison of SNPs and microsatellites as linkage mapping markers: Lessons from the zebra finch (Taeniopygia guttata). BMC Genomics 11, 218.

2. Berryers, T. G., Schmatz, S. M., Schimpf, R. J., Cowan, C. M., and Potter, J. (2003) TYRP1 is associated with dun coat colour in Dexter cattle or how now brown now? Anim. Genet. 34, 167-175.

3. Cheong, H. S., Kim, L. H., Namgoong, S., and Shin, H. D. (2013) Development of discrimination SNP markers for Hanwoo (Korean native cattle). Meat Sci. 94, 355-359.

4. Chung, E. R. and Chung, K. Y. (2004) Identification of beef breed using DNA marker of coat color genes. Korean J. Food Sci. An. 24, 355-360.

5. Chung, E. R., Kim. W. T., Kim, Y. S., and Han, S. K. (2000) Identification of Hanwoo meat using PCR-RFLP marker of MC1R gene associated with bovine coat color. Korean $J$. Anim. Sci. 42, 379-390.

6. Cone, R. D., Lu, D., Koppula, S., Vage, D. I., Kluagland, K., Boston, B., Chen, W., Orth, D. N., Pouton, C., and Kesterson, R. A. (1996) The melanocortin receptors: Agonists, antagonists, and the hormonal control of pigmentation. Recent. Prog. Horm. Res. 51, 287-317.

7. Do, K. T., Shin, H. Y., Lee, J. H., Kim, N. S., Park, E. W., Yoon, D. H., and Kim, K. S. (2007) Investigation of coat color candidate genes in Korean cattle (Hanwoo). Korean J. Anim. Sci. Technol. 49, 711-718.

8. Hong, J., Leem, D., Kim, M. G., Park, K. S., Yoon, T., No, K. M., and Jeong, J. (2010) Monitoring of restaurant beef labeling system. J. Food. Hyg. Safety 25, 162-169.

9. Hwang, E. G. (2010) A study on the effects of country of origin labelling and the traceability system for Hanwoo beef on safety and consumer trustworthiness. Korean J. Culinary Res. 16, 270-290.

10. Kim, J. B. and Lee, C. (2000) Historical look at the genetic improvement in Korean cattle: Review. Asian Australas. $J$. Anim. Sci. 13, 1467-1481.

11. Kim, S. W., Jang, H. K., Kim, K. S., Kim, J. J., Jeon, J. T., Yoon, D. H., Kang, S. H., Jung, H. I., and Cheong, I. C. (2009) Establishment of genetic characteristics and individual identification system using microsatellite loci in domestic beef cattle. J. Anim. Sci. Technol. 51, 273-282.

12. Korea Meat Trade Association (KMTA) (2011) Information and data of agricultural statistics of Korea. Accessed on January 12, 2011. http://kmta.or.kr/html/sub6-.html?scode=6.

13. Lee, H. K., Jeon, G. J., Kong, H. S., Oh, J. D., Choi, I. S., Kim, C. D., Jo, C. Y., Yoon, D. H., Shin, H. D., and Lee, J. H. (2004) Application of DNA test for individual traceability in Hanwoo (Korean Cattle). Korean J. Food. Sci. An. 24, 8-14.

14. Lee, K. H., Seo, K. S., Choi, T. J., Yun, D. H., Yang, D. Y., and Sang, B. C. (2008) Breeding and genetics: Comparison for genetic diversity between regional Hanwoo (Korean Cattle) brand group using microsatellite loci. Korean J. Anim. Sci. Technol. 50, 167-176.

15. Lim, D. G., Cha, J. S, Jo, C., Lee, K. H., Kim J. J., and Nam, K. C. (2014). Comparison of physicochemical and functional traits of Hanwoo steer beef by the quality grade. Korean $J$. Food Sci. An. 34, 287-296.

16. Lim, H. T., Min, H. S., Moon, W. G., Lee, J. B., Kim, J. H., 
Cho, I. C., Lee, H. K., Lee, Y. W., Lee, J. G., and Jeon, J. T. (2005) Analysis and selection of microsatellite for individual traceability system in Hanwoo. Korean J. Anim. Sci. Technol. 47, 491-500.

17. Lim, H. T., Seo, B. Y., Jung, E. J., Yoo, C. K., Yoon, D., and Jeon, J. T. (2009) A comparison of discriminating powers between 14 microsatellite markers and 60 SNP markers applicable to the cattle identification test. J. Anim. Sci. Technol. 51, 353-360.

18. MFDS (Ministry of Food and Drug Safety) (2013) Standards for processing and ingredients specifications of livestock products (MFDS notice 2013-244).

19. Min, B. R., Han, J. Y., and Lee, M. H. (1995) Animal products and processing; The identification of beef breeds (Korean cattle beef, holstein beef \& imported beef) using random amplified polymorphic DNAs. Korean J. Anim. Sci. Technol. 37, 651-660.

20. Mohanty, T. R., Seo, K. S., Park, K. M., Choi, T. J., Choe, H. S., Baik, D. H., and Hwang, I. H. (2008). Molecular variation in pigmentation genes contributing to coat colour in native Korean Hanwoo cattle. Anim. Genet. 39, 550-553.
21. Oh , J. D., Kong, H. K., Lee, J. H., Yang, D. T., Jeon, G. J., and Lee, H. K. (2008) Genetic variation and relationships of Korean cattle (Hanwoo) and foreign breeds using microsatellite markers. J. Anim. Sci. Technol. 50, 733-740.

22. Rannala, B. and Mountain, J. L. (1997) Detecting immigration by using multilocus genotypes. Proc. Natl. Acad. Sci. USA 94, 9197-9201.

23. Sasazaki, S., Hosokawa, D., Ishihara, R., Aihara, H., Oyama, K., and Mannen, H. (2011). Development of discrimination markers between Japanese domestic and imported beef. Animal Sci. J. 82, 67-72.

24. Van Eenennaam, A. L., Li, J., Thallman, R. M., Quaas, R. L., Dikeman, M. E., Gill, C. A., Franke, D. E., and Thomas, M. G. (2007). Validation of commercial DNA tests for quantitative beef quality traits. J. Animal Sci. 85, 891-900.

25. Yoon, D. H., Kong, H. S., Oh, J. D., Lee, J. H., Cho, B. W., Kim, J. D., Jeon, K. J., Jo, C., Jeon, G., and Lee, H. (2005) Establishment of an individual identification system based on microsatellite polymorphisms in Korea cattle (Hanwoo). Asian Australas. J. Anim. Sci. 18, 762-766.

(Received 2014.6.26/Revised 2014.10.8/Accepted 2014.10.12) 